\title{
Fatores relacionados à utilização de flumazenil em pacientes hospitalizados
}

\author{
Factors related to use of flumazenil in hospitalized patients \\ Factores relacionados con el uso de flumazenil en pacientes hospitalizados
}

\author{
Valcieny de Souza Sandes'; Guacira Correa Mattos ${ }^{I I} ;$ Rosany Bochner'II ; \\ Sônia Maria Cezar Góes ${ }^{I V}$; Elisangela da Costa Lima-Dellamora ${ }^{V}$
}

\begin{abstract}
RESUMO: Interações medicamentosas (IM) exacerbam o risco de eventos adversos graves relacionados ao uso de benzodiazepínicos. Este estudo objetivou conhecer aspectos relacionados à utilização de flumazenil em pacientes hospitalizados. Através do método observacional, foram analisados prescrições e prontuários de 31 pacientes internados entre junho/2008 e junho/2010 quanto à indicação do flumazenil, aos fatores que podem ter contribuído para intoxicação por benzodiazepínicos e à frequência de IM potenciais (IMP). A frequência de indicação para reversão de sedação excessiva foi aproximadamente 1,3 prescrições por 1.000 pacientes. Foram identificadas IMP em $84 \%$ dessas prescrições. Para sete casos, não houve prescrição prévia de benzodiazepínicos. O exame permitiu a identificação dos eventos com necessidade do manejo da sedação excessiva relacionados à ocorrência de IMP entre benzodiazepínicos e outros medicamentos para um elevado percentual de pacientes. Observou-se que idade elevada, quadro clínico com muitas comorbidades e administração de medicamentos com interações bem definidas estiveram associados à hipersedação.
\end{abstract}

Palavras-Chave: Flumazenil; interações de medicamentos; eventos adversos; benzodiazepínicos.

\begin{abstract}
Drug interactions (DIs) heighten the risk of severe adverse events connected with use of benzodiazepines. This observational study aimed to ascertain aspects of use of flumazenil in hospital inpatients. The prescriptions and medical records of 31 patients admitted between June 2008 and June 2010 were examined for indication of flumazenil, factors that could have contributed to benzodiazepine intoxication, and potential drug interaction (PDI) frequency. In 1.3 prescriptions per 1,000 patients the indication was for reversal of excessive sedation. PDIs were observed in $84 \%$ of these prescriptions. In 7 cases there was no prior prescription of benzodiazepines. The examination identified events requiring management of excessive sedation associated with occurrence of PDI between benzodiazepines and other drugs in a high percentage of patients. It was observed that more advanced age, clinical condition with many comorbidities, and administration of drugs with well-defined interactions were observed to associate with over-sedation.
\end{abstract}

Keywords: Flumazenil; drug interactions; adverse effects; benzodiazepines

RESUMEN: Interacciones medicamentosas (IM) incrementan el riesgo de efectos adversos graves. Este estudio observacional investigó los aspectos relacionados con el uso de flumazenil en los pacientes hospitalizados. Se analizaron las prescripciones y prontuarios de 31 pacientes ingresados entre junio/2008 y junio/2010. La frecuencia de la indicación de reversión de la sedación excesiva fue de 1,3 prescripciones por 1.000 pacientes. Se identificaron IMP en $84 \%$ de estas prescripciones. En siete casos no hubo prescripción previa de benzodiazepínicos. Este estudio permitió la identificación de la sedación excesiva asociada con la aparición de IMP entre los benzodiazepínicos y otras drogas para un alto porcentaje de pacientes. Se ha observado que la edad avanzada, cuadro clínico con muchas comorbilidades y administración de fármacos con interacciones bien definidas se asociaron con excesiva sedación.

Palabras Clave: Flumazenil; interacciones medicamentosas; efectos adversos; benzodiazepínicos.

\section{INTRODUÇÃO}

O risco na utilização de medicamentos em pacientes hospitalizados bem como as implicações quanto aos erros no processo de cuidado vem ganhando amplo destaque na literatura ${ }^{1,2}$. No entanto, apesar da gravidade do problema, o desenvolvimento e adoção de práticas seguras pela equipe de saúde durante a formação e atuação profissional são prejudicados pela falta de padronização de conceitos além de subnotificação sobre a ocorrência de eventos adversos e seus fatores causais ${ }^{3}$.

\footnotetext{
'Farmacêutica, Especialista em Farmácia Hospitalar, Programa de Pós-Graduação em Farmácia Hospitalar, Faculdade de Farmácia, Universidade Federal Fluminense. Niterói, Brasil. E-mail: valcienyfar@hotmail.com.

IIFarmacêutica, Doutora em Saúde Pública, Professora Adjunta da Faculdade de Farmácia, Universidade Federal do Rio de Janeiro. Brasil. E-mail: gcmatos@globo.com.

IIIEstatística, Doutora em Saúde Pública, Tecnologista em Saúde Pública da Fundação Oswaldo Cruz. Rio de Janeiro, Brasil. E-mail: rosany@icict.fiocruz.br.

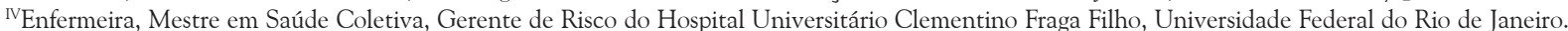
Brasil.E-mail: sonia.cezar.goes@gmail.com.

vFarmacêutica, Doutora em Saúde Pública, Professora Adjunta da Faculdade de Farmácia, Universidade Federal do Rio de Janeiro. Brasil. E-mail: lima.dellamora@gmail.com.
} 
Os eventos adversos a medicamentos (EAM) provocam agravos à saúde dos pacientes acarretando danos permanentes, prolongando o tempo de internação e aumentando custos com saúde. Métodos de monitoramento e prevenção desses eventos têm sido avaliados e utilizados como indicadores de qualidade em sistemas sanitários ${ }^{4,5}$.

Sabe-se que o uso simultâneo de vários medicamentos é comum na prática clínica e está intrinsecamente relacionado ao risco de interações medicamentosas e reações adversas ${ }^{6}$. Dentre os medicamentos comumente envolvidos em eventos adversos, destacam-se os psicotrópicos? ${ }^{7}$. Sobre as formulações com esta ação, os benzodiazepínicos são utilizados como hipnóticos de escolha, em função de sua eficácia e segurança, estando entre os fármacos mais consumidos no mundo ${ }^{8}$.

No Brasil, o consumo exagerado de benzodiazepínicos pode ser inferido pelo fato dessa classe terapêutica ser responsável pelo maior número de intoxicações. Segundo dados do Centro de Informação Toxicológica do Rio Grande do Sul, em 2009, foram registrados 9.271 casos de intoxicação por medicamentos, sendo que 220 ocorreram, de forma não intencional, por benzodiazepínicos ${ }^{9}$.

A detecção e a documentação de eventos adversos a benzodiazepínicos são difíceis. No entanto, o estudo do uso de flumazenil pode ser útil para sinalizar a ocorrência e estimar a frequência de sedação excessiva por essa classe terapêutica ${ }^{10,11}$. Por se tratar de fármacos com ação no sistema nervoso central, o conhecimento sobre o uso de flumazenil é particularmente importante, tanto no que tange à análise do perfil de uso em um ambiente de formação de profissionais de saúde e à identificação de eventos adversos e erros de medicação envolvidos, quanto na adoção de medidas que garantam a segurança do paciente. Estima-se que, no Brasil, ocorram 4.800.000 novos casos de intoxicação acidental ou intencional por ano, onde 0,1 a 0,4\% resultam em óbito ${ }^{12}$. Apesar de muitos estudos abordarem a intoxicação intencional com psicotrópicos, a investigação sobre a frequência do uso de flumazenil em pacientes hospitalizados é escassa no país.

Objetivo do presente trabalho foi conhecer os aspectos e elementos relacionados à utilização de flumazenil em pacientes internados em um hospital geral universitário e discutir fatores, grupos de maior risco e situações clínicas que propiciaram a sedação excessiva e consequente uso do referido antídoto.

\section{REVISÃo DE LITERATURA}

A intoxicação por benzodiazepínicos pode causar depressão do sistema nervoso central, com variado grau de queda do nível de consciência, sonolência, letargia, sedação excessiva, confusão mental, ataxia, amnésia, depressão respiratória, hipotensão, hipotermia, bradicardia e coma, além de estar associado ao risco de quedas em idosos. É comum a exacerbação dos efeitos terapêuticos na coadministração de benzodiazepínicos com outros medicamentos, pelo potencial risco de interações medicamentosas que influenciam a farmacocinética dos primeiros e aumentam a sua toxicidade ${ }^{8}$.

O fármaco flumazenil (8-fluoro-5,6-diidro5-metil-6-oxo-4H-imidazol [1,5a] [1,4] benzodiazepina - 3-carboxilato de etila) bloqueia, por inibição competitiva, os efeitos centrais de substâncias que agem ao nível dos receptores benzodiazepínicos ${ }^{13}$. É classificado como antídoto pela Anatomical Therapeutic Chemical (ATC) sob o código V03AB25. Não possui dose diária definida (DDD) e seu uso é indicado, principalmente, na reversão da ação sedativa após a anestesia, bem como no tratamento da superdosagem aguda de benzodiazepínicos ${ }^{14,15}$. Outros usos, ainda que insuficientemente estabelecidos, foram descritos na literatura para melhora do estado mental de pacientes com hepatopatias graves (encefalopatia hepática $)^{16,17}$, intoxicação alcoólica ${ }^{18,19}$ e intoxicação por plantas do gênero Cannabis ${ }^{20}$.

\section{Metodologia}

Foi realizado um estudo observacional de caráter exploratório, descritivo e retrospectivo. Foram analisados dados de pacientes hospitalizados, no período de junho de 2008 a junho de 2010, de um hospital público federal, do tipo geral e de grande porte, localizado no Estado do Rio de Janeiro e cadastrado para atenção de alta complexidade para demanda referenciada, sem atendimento de emergência ${ }^{21}$.

Foram incluídos no estudo todos os pacientes internados em leitos clínicos com idade igual ou superior a 18 anos, para os quais foi necessária a prescrição de flumazenil no período de investigação. Obteve-se a relação de pacientes na condição especificada, a partir de consulta ao sistema informatizado de prescrição e prontuário eletrônico da instituição. Excluíram-se os pacientes em observação pós-operatória devido à rotina de utilização deste fármaco para reversão da sedação do procedimento anestésico ${ }^{15}$.

Entre os 19.355 internados no hospital durante o período investigado, 31 utilizaram o antídoto flumazenil e foram selecionados para o estudo. A priori, foram coletados dados, em prontuário médico informatizado, referentes ao perfil farmacoterapêutico, complementados pelo histórico clínico, comorbidades diagnosticadas, exames e procedimentos realizados, sexo e idade dos pacientes.

Os medicamentos foram classificados até o quinto nível, segundo o método ATC indicado pela 
Organização Mundial de Saúde ${ }^{14}$. As informações, organizadas em planilha eletrônica, foram analisadas pela abordagem quantiqualitativa. Buscou-se investigar: a indicação do flumazenil; os fatores que podem ter contribuído para o estado de intoxicação pelos benzodiazepínicos e a frequência de interações medicamentosas potenciais entre benzodiazepínicos e outros medicamentos prescritos durante a internação dos pacientes estudados.

Para a análise qualitativa do uso de flumazenil, elaborou-se uma matriz teórico-conceitual fundamentada em uma revisão integrativa da literatura quanto à indicação do medicamento ${ }^{22}$. Utilizou-se as bases de dados eletrônicas MEDLINE, LILACS, SCIELO a partir da Biblioteca Virtual de Saúde (BVS) para a busca de estudos indexados em português, inglês e espanhol entre os anos de 1990 e 2010. A base Micromedex ${ }^{\circledR}$ foi utilizada como referência para identificação e classificação de severidade das interações ${ }^{23}$.

Obteve-se a prevalência como medida de frequência, calculada a partir do número de casos observados sobre o número total de pacientes internados no período.

O estudo foi submetido e aprovado pelo Comitê de Ética em Pesquisa da instituição, onde foi realizado, sob parecer $\mathrm{n}^{\circ} 742 / 10$ em 22 de setembro de 2010.

\section{Resultados e Discussão}

\section{Fatores associados à prescrição e uso de flumazenil}

O tempo de internação dos 31 pacientes selecionados no estudo variou entre 8 e 300 dias, com média igual a 57 e mediana 37,5 dias. A maioria foi do sexo masculino (53\%) e possuía mais de 60 anos (56,2\%). A variação de idade foi entre 23 e 82 anos, com média de $59 \pm 19$ anos e mediana de 64 anos. As principais doenças e comorbidades associadas que estavam relatadas no prontuário são apresentadas na Figura 1.
Para grande parte dos pacientes, a dose prescrita de flumazenil foi de 0,5 miligramas em até 24 horas após o uso do benzodiazepínico. Contudo, houve situações em que este período se estendeu por até 4 dias.

O flumazenil foi indicado para o tratamento de sedação excessiva em 24 pacientes, representando uma frequência aproximada de 1,3 prescrições por 1000 pacientes. Nos demais casos (sete), seu uso ocorreu sem o registro de prescrição prévia de benzodiazepínicos. Entre estes últimos, em quatro prontuários encontrouse registrado o teste do flumazenil como indicação de uso, a qual se apoiava na suspeita de intoxicação por benzodiazepínicos anteriormente à internação. No caso de um paciente, havia menção, no prontuário, de exacerbação do efeito da morfina, sugerindo confusão entre a indicação dos antídotos naloxona e flumazenil para reversão da ação de opióides e benzodiazepínicos, respectivamente. Em outras duas ocorrências, não foi identificado o motivo de uso, não sendo possível, contudo, descartar a prescrição de benzodiazepínicos para procedimentos não registrados em prontuário.

Para 84\% dos pacientes, para os quais foi necessária a prescrição de flumazenil após o uso de benzodiazepínicos, observou-se a ocorrência de interações medicamentosas potenciais (IMP) devido a associações entre estes sedativos e outros medicamentos utilizados, durante o período de hospitalização. A análise do histórico registrado em prontuário destes pacientes permitiu a identificação de fatores concorrentes, já referidos na literatura, como: idade; condições de saúde e comprometimento da função renal e hepática, que podem ter influenciado a suscetibilidade para interações medicamentosas. Tais fatores, bem como os medicamentos envolvidos em cada caso, estão descritos na Tabela 1.

\section{Frequência de interações medicamentosas potenciais}

Os benzodiazepínicos prescritos pertenciam à lista de medicamentos essenciais do hospital investigado e possuíam atividade de longa (diazepam, clonazepam) e curta duração (midazolam). Estes foram administrados

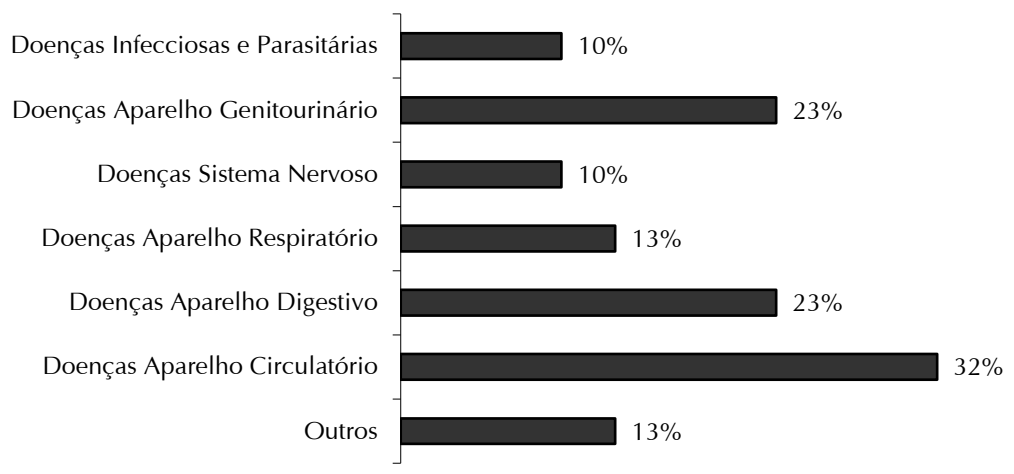

FIGURA 1: Distribuição percentual das principais doenças na população estudada. Rio de Janeiro, 2010. $(\mathrm{N}=31)$ 
TABELA 1: Descrição dos casos e das interações medicamentosas potenciais moderadas ou graves entre benzodiazepínicos e outros medicamentos identificados. Rio de Janeiro, 2010. ( $N=20)$

\begin{tabular}{|c|c|c|c|c|c|}
\hline Caso & Sexo & Idade & $\begin{array}{c}\text { Comorbidades associadas ao } \\
\text { quadro clínico }\end{array}$ & $\begin{array}{c}\text { Benzo-diazepínicos } \\
\text { utilizados }\end{array}$ & $\begin{array}{l}\text { Medicamentos envolvidos } \\
\text { (classificação ATC) }\end{array}$ \\
\hline 1 & $\mathrm{~F}$ & 23 & Epilepsia e AVC & Diazepam & Omeprazol (A02BC) \\
\hline 2 & $\mathrm{~F}$ & 68 & $\begin{array}{l}\text { Cirrose, fratura do colo do fêmur, } \\
\text { encefalopatia hepática }\end{array}$ & Diazepam & Codeína (N02AA) \\
\hline 3 & M & 25 & Fratura de Costela e Distonia & & \\
\hline 4 & $\mathrm{~F}$ & 80 & $\begin{array}{l}\text { Crise convulsiva parcial, } \\
\text { menangiomarecindivante }\end{array}$ & Diazepam & $\begin{array}{l}\text { Omeprazol (A02BC) } \\
\text { Fenobarbital (N03AA) }\end{array}$ \\
\hline 5 & M & 35 & Cardiopatia, fibrilação atrial & Diazepam & Ranitidina (A02BA) \\
\hline 6 & $\mathrm{~F}$ & 71 & $\begin{array}{l}\text { HAS, fibrilação atrial e hepatopatia } \\
\text { congestiva }\end{array}$ & Midazolam & \\
\hline 7 & M & 81 & $\begin{array}{l}\text { Mielodisplasia, sepse, edema } \\
\text { agudo pulmonar }\end{array}$ & $\begin{array}{l}\text { Diazepam } \\
\text { Midazolam }\end{array}$ & Omeprazol (A02BC) \\
\hline 8 & $\mathrm{~F}$ & 61 & $\begin{array}{l}\text { Pneumectomia esquerda, HAS, } \\
\text { crise convulsiva }\end{array}$ & & \\
\hline 9 & $\mathrm{~F}$ & 50 & $\begin{array}{l}\text { Insuficiência cardíaca congestiva, } \\
\text { HAS, DM }\end{array}$ & & \\
\hline 10 & M & 51 & $\begin{array}{l}\text { Hepatite C, cirrose, gastropatia } \\
\text { hipertensiva portal, encefalopatia } \\
\text { hepática }\end{array}$ & Midazolam & Omeprazol (A02BA) \\
\hline 11 & M & 58 & DM, Insuficiência Renal Crônica & & \\
\hline 12 & $\mathrm{~F}$ & 52 & $\begin{array}{l}\text { Doença mitral reumática, } \\
\text { Fibrilação atrial }\end{array}$ & & \\
\hline 13 & $\mathrm{~F}$ & 71 & $\begin{array}{l}\text { Diabetes insípidus, DM, trombose } \\
\text { venosa profunda }\end{array}$ & & \\
\hline 14 & M & 75 & HAS, DM, hipotireoidismo & & \\
\hline 15 & M & 55 & Adenocarcinoma de cólon & Midazolam & Ranitidina (A02BA) \\
\hline 16 & $\mathrm{~F}$ & 78 & $\begin{array}{l}\text { Cardiomiopatia, endocardite, } \\
\text { choque séptico }\end{array}$ & Midazolam & $\begin{array}{l}\text { Ranitidina (A02BA) } \\
\text { Morfina (N02AA) }\end{array}$ \\
\hline 17 & M & 74 & $\begin{array}{l}\text { Acidente com animal peçonhento, } \\
\text { celulite em membro superior } \\
\text { direito, insuficiência renal }\end{array}$ & Midazolam & $\begin{array}{l}\text { Fluconazol (J02AC) } \\
\text { Ranitidina (A02BA) }\end{array}$ \\
\hline 18 & $\mathrm{~F}$ & 60 & $\begin{array}{l}\text { Doença pulmonar obstrutiva } \\
\text { crônica, hemorragia no trato } \\
\text { gastrointestinal, } \\
\text { hiperparatireoidismo }\end{array}$ & $\begin{array}{l}\text { Midazolam } \\
\text { Diazepam }\end{array}$ & $\begin{array}{l}\text { Fluconazol (J02AC) } \\
\text { Ranitidina (A02BA) }\end{array}$ \\
\hline 19 & M & 43 & $\begin{array}{l}\text { Infecção por HIV } \\
\text { Infecção por Paracoccidiomicose }\end{array}$ & $\begin{array}{l}\text { Midazolam } \\
\text { Diazepam }\end{array}$ & $\begin{array}{l}\text { Efavirenz (J05AG) } \\
\text { Ranitidina (A02BA) }\end{array}$ \\
\hline 20 & M & 66 & HIV, doença renal crônica & Midazolam & Ritonavir (J05AE) \\
\hline
\end{tabular}

em doses terapêuticas para a realização de procedimentos como intubação traqueal, cardioversão elétrica e punção, ou, ainda, para manutenção do paciente em sedação.

A prescrição do benzodiazepínico em decorrência de crise convulsiva ocorreu em quatro casos, sendo que dois destes pacientes eram epilépticos. Em situações de indução anestésica observou-se a associação de dois benzodiazepínicos.

Foram encontradas interações farmacológicas de leve a grave nas prescrições de 20 pacientes, algumas com até três interações concomitantes. A gravidade, frequência e descrição dos efeitos farmacológicos das 36 interações potenciais encontradas foram organizadas na Tabela 2.
Apenas nos casos de 12 pacientes, os eventos decorrentes do uso dos benzodiazepínicos estavam identificados e descritos no prontuário médico como sedação excessiva com ou sem redução do nível de consciência (9), hipotensão (1), desorientação (1) e depressão respiratória (1).

O flumazenil não é um medicamento utilizado de forma rotineira na maioria das instituições hospitalares. É indicado para casos de suspeita de superdosagem por benzodiazepínicos e reversão dos efeitos sedativos. A literatura o descreve como um rastreador específico para a detecção de eventos adversos a medicamentos ${ }^{24}$, o que assinala a relevância de se investigar seu uso. 
TABELA 2: Classificação, frequência e descrição das interações medicamentosas potenciais observadas. Rio de Janeiro, 2010. $(\mathrm{N}=36)$

\begin{tabular}{|c|c|c|c|c|}
\hline $\begin{array}{c}\text { Benzo- } \\
\text { diazepínico }\end{array}$ & $\begin{array}{c}\text { Medicamentos } \\
\text { envolvidos }\end{array}$ & Classificação & Frequência & Descrição da interação(*) \\
\hline \multirow{4}{*}{ Diazepam } & Codeína & Grave & 2 & Efeito aditivo da depressão respiratória \\
\hline & z & Grave & 2 & Efeito aditivo da depressão respiratória \\
\hline & Omeprazol & Leve & 6 & $\begin{array}{l}\text { Exacerbação e prolongamento do efeito } \\
\text { do diazepam }\end{array}$ \\
\hline & Fenitoína & Moderada & 2 & $\begin{array}{l}\text { Pode causar alterações na concentração } \\
\text { da fenitoína }\end{array}$ \\
\hline \multirow{7}{*}{ Midazolam } & Enfavirenz & Contra-indicado & 1 & $\begin{array}{l}\text { Aumento do risco de toxicidade do } \\
\text { midazolam }\end{array}$ \\
\hline & Fluconazol & Moderada & 2 & $\begin{array}{l}\text { Aumento da concentração plasmática do } \\
\text { midazolam }\end{array}$ \\
\hline & $\begin{array}{l}\text { Lopinavir + } \\
\text { Ritonavir }\end{array}$ & Contra-indicado & 1 & $\begin{array}{l}\text { Aumento da concentração plasmática do } \\
\text { midazolam }\end{array}$ \\
\hline & Morfina & Grave & 1 & Efeito aditivo da depressão respiratória \\
\hline & Omeprazol & Moderada & 8 & Aumento da toxicidade do midazolam \\
\hline & Ranitidina & Moderada & 7 & $\begin{array}{l}\text { Aumento da biodisponibilidade do } \\
\text { midazolam }\end{array}$ \\
\hline & Fenitoína & Moderada & 4 & $\begin{array}{l}\text { Pode resultar em redução da eficácia do } \\
\text { midazolam }\end{array}$ \\
\hline
\end{tabular}

Um estudo analisou os agravos provocados por todos os tipos de medicamentos - efeitos adversos, intoxicações, erros na prescrição ou na administração, envenenamentos e lesões autoprovocadas - em pacientes internados pelo Sistema Único de Saúde (SUS) no Estado do Rio de Janeiro, observando a prevalência de 1,8 agravos por 1.000 internações ${ }^{25}$, muito aproximada à encontrada nesta pesquisa, que foi de 1,3 por 1.000. Contudo, os dados do presente estudo destacam-se por excluir os casos relativos às intoxicações intencionais provocadas por benzodiazepínicos, cuja frequência é bastante significativa ${ }^{9}$, ao focar, especificamente, os eventos ocorridos pelo uso terapêutico de medicamentos durante a internação em um hospital universitário, que não possui atendimento de emergência aberto à população geral.

A prescrição de flumazenil deve ser cautelosa e realizada como conduta estratégica quando, sabidamente, a overdose é devida a benzodiazepínicos ${ }^{8}$. Sua administração empírica é questionada em pacientes comatosos devido à ocorrência de eventos adversos que incluem: arritmia cardíaca, dor no local da administração, vertigem, náuseas, visão anormal ou borrada, agitação, irritabilidade e ressedação ${ }^{23}$.

A investigação do histórico clínico e medicamentoso dos pacientes possibilitou a observação de outras discordâncias em seu uso. Em um caso, o flumazenil foi prescrito para intoxicação pelo uso de morfina, conforme relatado em prontuário médico, o que leva a suposição de que este medicamento foi confundido com naloxona, fármaco preconizado para intoxicação por opióides. Tal evento caracteriza-se como um erro de medicação do tipo erro de prescrição, pela possi- bilidade de causar ou induzir ao uso inapropriado de medicamento e por ser evitável ${ }^{26,27}$.

A administração de flumazenil é contraindicada em pacientes com aumento de pressão intracraniana e com história de epilepsia ${ }^{8}$. No presente estudo, 4 $(12,5 \%)$ pacientes haviam utilizado benzodiazepínicos para o tratamento de convulsões, duas delas relacionadas ao diagnóstico de epilepsia grave, segundo relato clínico.

Uma análise de 43 casos de convulsões após o uso de flumazenil, realizada em outro estudo, demonstrou que a causa das mesmas esteve relacionada à associação com antidepressivos tricíclicos ou desmascaramento do efeito anticonvulsivo dos benzodiazepínicos ${ }^{28}$. Ensaios clínicos indicaram que o risco de óbito é alto na administração de flumazenil em pacientes que utilizam antidepressivos tricíclicos em altas doses ou possuem graves doenças ${ }^{8,23}$.

Quanto às interações medicamentosas potenciais em exame, a maioria foi de moderada a alta severidade. Estas estiveram associadas a quadros clínicos complexos. Merece destaque o fato de que a biotransformação dos benzodiazepínicos de longa duração e da maioria dos de ação ultracurta está sujeita a variações individuais nos parâmetros farmacocinéticos, atribuídos a fatores como idade, dano hepático, e uso concomitante de indutores e inibidores enzimáticos ${ }^{8,10}$. Os benzodiazepínicos devem ser evitados em pacientes idosos pela particular sensibilidade deste grupo etário devida à redução do fluxo sanguíneo hepático e à redução da atividade enzimática ${ }^{29,30}$. A combinação do declínio fisiológico com o aumento de comorbidades pode levar ao comprometimento cognitivo, delírios, 
quedas e fraturas $^{31}$. Os resultados do presente estudo refletem este risco, uma vez que foram observados pacientes cujas doenças —insuficiência cardíaca, renal e hepática - influenciaram a farmacocinética dos benzodiazepínicos, além da instabilidade hemodinâmica relacionada às outras patologias e à gravidade do estado clínico.

A ocorrência de fraturas graves decorrentes de queda em idosos brasileiros que utilizam benzodiazepínicos é bem conhecida na literatura ${ }^{32}$. A hipersedação exacerba este risco. A prevenção das quedas é uma das ações previstas pelo recente Programa Nacional de Segurança do Paciente (PNSP) ${ }^{33}$. O protocolo de prevenção de quedas que integra o PNSP reafirma a necessidade de atenção por parte da equipe de enfermagem quanto às classes de medicamentos utilizados pelo paciente idoso ${ }^{33}$. A interação com o farmacêutico clínico é recomendada e contribui para a cooperação e troca de saberes entre a equipe multidisciplinar no que tange ao processo de racionalização da terapêutica ${ }^{33,34}$.

Pesquisadores relataram que o risco de lesão na população dobra quanto existe associação entre benzodiazepínicos e outros medicamentos ${ }^{35}$. Todavia, o fato de os benzodiazepínicos serem reconhecidos como medicamentos bem tolerados $^{8} \mathrm{e}$ a existência de um medicamento para reversão de seus efeitos pode contribuir para que outros riscos no seu uso sejam desconsiderados no cotidiano hospitalar.

Por este motivo, a associação de depressores do sistema nervoso central em idosos ou em pacientes com insuficiência renal, cardíaca ou hepática deve ser monitorada, sendo indicado o uso de doses menores que as usuais. Os efeitos sobre o sistema cardiovascular são geralmente provocados por diminuição da resistência vascular periférica, depressão miocárdica e redução do débito cardíaco ${ }^{36}$.

$\mathrm{O}$ uso de flumazenil esteve arrolado, em maior grau, à administração prévia de midazolam, sedativo extensamente utilizado no ambiente hospitalar. $O$ fato de este benzodiazepínico estar envolvido na maioria das situações que demandaram reversão da sedação condiz com sua classificação como medicamento potencialmente perigoso ${ }^{37}$. Comparando-o aos demais medicamentos da mesma classe, o midazolam possui importante variabilidade farmacocinética intraindividual $^{38}$. Considerando-se a ocorrência de interações medicamentosas potenciais em $84 \%$ dos casos, cabe ressaltar que muitas situações clínicas requerem, frequentemente, o uso de medicamentos sabidamente conflitantes, o que torna complexa a prevenção de possíveis eventos adversos ${ }^{10}$. Suspeita-se que oflumazenil seja a principal alternativa para impedir ou minimizar o dano desses eventos, em detrimento de outras estratégias como administração de doses menores e rigoroso monitoramento clínico. Um estudo realizado em um hospital paulista, também universitário, sobre o uso de midazolam, relatou que em $88 \%$ dos casos, onde foi necessária a prescrição de flumazenil, ocorreram interações medicamentosas clinicamente significantes entre benzodiazepínicos e agonistas opiáceos, furosemida, pancurônio e gluconato de cálcio ${ }^{10}$.

No presente trabalho, destacaram-se as interações com medicamentos utilizados no tratamento de úlceras pépticas e refluxo gastroesofágico e os antirretrovirais, pela frequência observada e contraindicação da associação, respectivamente. É possível que a rotineira, e por vezes inapropriada, prescrição de omeprazol e ranitidina para os pacientes internados seja o motivo da frequência observada ${ }^{39}$.

A necessidade de precaução pelo risco de intoxicação foi reafirmada em um recente trabalho sobre a farmacodinâmica de medicamentos utilizados para sedação profunda ${ }^{30}$, e especialistas também reforçam a importância do acompanhamento multiprofissional desses casos com a participação ativa ${ }^{2}$. A vigilância e análise cuidadosa de falhas durante a terapia medicamentosa pelas instituições devem contribuir para a sua diminuição $0^{2,40}$. Alguns esforços para reduzir o uso inapropriado de benzodiazepínicos já são relatados ${ }^{41}$ e incluem abordagens educacionais, de alertas, de auditoria e feedback. Esses autores reforçam que a interação multiprofissional entre prescritores, farmacêuticos e enfermeiros é de fundamental importância na melhoria da segurança dos pacientes.

\section{Conclusão}

Por ter sido realizado exclusivamente com a população de um hospital do Estado do Rio de Janeiro, este estudo possui a limitação de não comportar generalizações. A análise realizada, contudo, permitiu a identificação e a necessidade de manejo da sedação excessiva - reação adversa severa e com importantes complicações clínicas e econômicas — relacionada à ocorrência de interações medicamentosas potenciais entre benzodiazepínicos e outros medicamentos para um elevado percentual de pacientes.

Os resultados apontaram que a presença de fatores como: idade elevada, quadro clínico com muitas comorbidades e administração de medicamentos com interações bem definidas, bem como suas características, estiveram associados, na maioria dos casos analisados, ao uso do antídoto flumazenil para reversão da sedação.

Nesse sentido, o fato de esta reação ser prevenível sugere que o hospital de ensino estudado carece de maior sensibilização da equipe multiprofissional quanto às estratégias para segurança do paciente e os riscos da terapia medicamentosa. A observação de discordâncias no uso de flumazenil reforça a necessidade de se ampliar a produção de conhecimento sobre o tema. 
$\mathrm{O}$ aporte metodológico dos estudos de utilização de medicamentos (um fármaco indicado como antídoto na presente investigação) configurou-se como uma preciosa ferramenta para a observação e a identificação de eventos associados ao uso dos benzodiazepínicos durante o processo de cuidado em saúde. A severidade e os efeitos decorrentes destas interações mostraram-se relevantes, o que incita a realização de novos estudos sobre o uso de medicamentos com ação no sistema nervoso central, bem como o rigoroso monitoramento terapêutico.

\section{REFERÊNCIAS}

1.Rosa MB, Perini E, Anacleto TA, Neiva HM, Bogutchi T. Erros na prescrição hospitalar de medicamentos potencialmente perigosos. Rev Saúde Pública. 2009; 43: 490-8. 2.Lopes DMA, Néri EDR, Madeira LS, Souza Neto PJ, Lélis ARA, Souza TR, et al. Análise da rotulagem de medicamentos semelhantes: potenciais erros de medicação. Rev Assoc Med Bras. 2012; 58(1): 95-103.

3.Silva AEBC. Segurança do paciente: desafios para a prática e a investigação em enfermagem. Rev Eletr Enf. 2010; 12: 422.

4.Naessens JM, Campbell CR, Huddleston JM, Berg BP, Lefante JJ, Williams AR, Culbertson RA. A comparison of hospital adverse events identified by three widely use detection methods. Int J Qual Health Care. 2009; 21: 301-7. 5.Souza LP, Bezerra ALQ, Silva AEC, Carneiro FS, Paranaguá TTB, Lemos LF. Eventos adversos: instrumento de avaliação do desempenho em centro cirúrgico de um hospital universitário. Rev enferm UERJ. 2011; 19: 127-33. 6.Secoli SR. Polifarmácia: interações e reações adversas no uso de medicamentos por idosos. Rev Bras Enferm. 2010; 63: 136-40.B

7.Forster AJ, Halil RB, Tierney MG. Pharmacist surveillance of adverse drug events.Am J Health-System Pharm. 2004; 61:1466-72.

8.Oga S, Camargo MMA, Batistuzzo JAO. Fundamentos da toxicologia. $3^{\text {a }}$ ed. São Paulo: Atheneu; 2008.

9.Centro de Informação Toxicológica do Rio Grande do Sul. Secretaria Estadual de Saúde. ToxicovigilânciaToxicologia Clínica: dados e indicadores selecionados - 2009/2010. Porto Alegre (RS): CIT-RS; 2011.

10.Kawano FD, Ueta J, Sankarankutty AK, Pereira LRL, Freitas O. Midazolam-related drug interactions: detection of risk situations to the patient safety in a Brazilian teaching hospital. J Patient Saf. 2009; 5(2): 69-74.

11.Naessens JM, O'byrne TJ, Johnson MG, Vansuch MB, Mcglone CM, Huddleston JM. Measuring hospital adverse events: assessing inter-rater reliability and trigger performance of the Global Trigger Tool. Int J Qual Health Care. 2010; 22: 266-74.

12.Zambolim CM, Oliveira TP, Hoffmann NA, Vilela CEB, Neves D, Anjos FR, et al. Perfil das intoxicações exógenas em um hospital universitário. Rev Med Minas Gerais. 2008; 18(1): 5-10.

13.Karavokiros KA, Tsipis GB. Flumazenil: a benzodiazepineantagonist. DICP. 1990; 24:976-81.
14.WHOCC - World Health Organization Collaborating Centre for Drug Statistics Methodology. [site de Internet] [cited in 2014 Apr 12]. Disposable in: www.whocc.no/ 15.Agencia Nacional de Vigilãncia Sanitária. Flumazenil. [citado em 15 fev 2014]. Disponível em: www.anvisa.gov. br/base/visadoc/BM/BM[26097-1-0].

16.Als-Nielsen B, Gluud LL, Gluud C. Benzodiazepine receptor antagonists for hepatic encephalopathy. Cochrane database Syst Rev. 2001; (4): CD002798.

17.Kuromashiro R. Treatment of minimal hepatic encephalopathy. Hepatol Res. 2008; (38): S128-31.

18.Anton RF, Myrick H, Baros AM, Latham PK, Randall PK, Wright TM et al. Efficacy of a combination of flumazenil and gabapentin in the treatment of alcohol dependence: relationship to alcohol withdrawal symptoms. J Clin Psycopharmacol. 2009; 29: 334-42.

19.Soyka M, Rösner S. Emerging drugs to treat alcoholism. Expert Opin Emerg Drugs. 2010;15 :695-711.

20.Lassaletta A, Martino R, Gónzalez-Santiago P, Torrijos C, Cebrero M, García-Frías E. Reversal of na antihistamine-induced coma with flumazenil. Pediatr Emerg Care. 2004; 20: 319-20.

21.Cadastro Nacional de Estabelecimentos de Saúde (Br). [site de Internet] [citado em 25 jun 2011]. Disponível em: www.cnes.datasus.gov.br.

22. Castro CGSO. Estudos de utilização de medicamentos: noções básicas. Rio de Janeiro: Fiocruz; 2000.

23.Micromedex ${ }^{\circledR}$ Healthcare Series [site de Internet]. Greenwood Village, Colo: Thomson Reuters (Healthcare) Inc. Updatedperiodically. [cited in 2014 Jun 02]. Disponsable in: http://www.micromedex.com

24.Rozich JD, Haraden CR, Resar RK. Adverse drug event trigger tool: a pratical methodology for measuring medication related harm. Qual Saf Health Care. 2003; 12: 194-200. 25.Rozenfeld S. Agravos provocados por medicamentos em hospitais do Estado do Rio de Janeiro, Brasil. Rev SaudePublica. 2007; 41: 108-15.

26.Silva AEBC, Cassiani SHBC. Erros de medicação em uym hospital universitário: tipo, causas, sugestões e providências. Rev Bras Enferm. 2004; 57:671-4.B

27.National Coordinating Council for Medication Error Reporting and Prevention (NCC MERP). Whatis a medicationerror; 1998. [site de Internet]. [cited in 2011 nov 12] Disponsable in: http://www.nccmerp.org/medErrorTaxonomy.html.

28.Spivey WH. Flumazenil and seizures: analysis of 43 cases. Clin Ther. 1992; 14: 292-305.

29.Fick DM, Cooper JW, Wade WE, Waller JL, Maclean JR, Beers MH. Updating the beers criteria for potentially inappropriate medication use in older adults results of a US consensus panel of experts. Arch Intern Med. 2003;163:2716-25.

30.Becker DE. Pharmacodynamic considerations for moderate and deep sedation. Anesth Prog.2012; 59:28-42. 31.Cresswell KM, Fernando B, McKinstry B, Sheikh A. Adverse drug events in the elderly. British Medical Bulletin.2007; 83: 259-74.

32.Coutinho ESF, Silva SD. Uso de medicamentos como fator de risco para fratura grave decorrente de queda em idosos. Cad Saúde Pública. 2002; 18:1359-66. 
33.Ministério da Saúde (Br). Portaria no 529, de 1ํ de abril de 2013. Institui o Programa Nacional de Segurança do Paciente (PNSP). Brasília (DF): Gabinete Ministerial; 2013. 34.Matos E, Pires DEP, Gelbcke FL. Implicações da interdisciplinaridade na organização do trabalho da enfermagem: estudo em equipe de cuidados paliativos. Rev Eletr Enf.[Internet]. 2012;14:230-9

35.French DD, Chirikos TN, Spehar A, Campbell R, Means H, Bulat T. Effect of concomitant use of benzodiazepines and other drugs on the risk of injury in a veterans population. Drug Safety. 2005; 28:1141-50.

36.Cury AF, Vieira MLC, Fischer CH, Rodrigues ACT, Cordovil A, Monaco C et al. Segurança da ecocardiografiatransesofágicaemadultos. Estudoem um hospital multidisciplinar. Arq Bras Cardiol 2009;93:478-83.

37.ISMP. Institute for safe medication practices. [site de
Internet]. [cited in 2014 Jun 10]. Disposable in: www. ismp.org.

38.Swart EL, Zuideveld KP, Jongh J, Danhof M, Thijs TG, Schijndel RMJS. Comparative population pharmacokinetics of lorazepam and midazolam during long-term continuous infusion in critically ill patients. Br J Clin Pharmacol. 2004; 57:2 135-45.

39.Munari L, Hart D, Morrone FB. Uso de omeprazol em hospital universitário de Porto Alegre-RS (Brasil). Seguimiento Farmacoterapéutico. 2004; 2: 235-43

40.Rosa EB, Perini E. Erros de medicação: Quem foi? Rev Assoc Med Bras. 2003; 49: 335-41

41.Smith AJ, Tett SE. Improving the use of benzodiazepines - is it possible? A non-systematic review of interventions tried in the last 20 years. BMC Health Serv Res. 2010; 10: 321. 